\section{Tinea incognito: Clinical perspectives of a new imitator}

\author{
Samer Dhaher \\ Head of Department of Dermatology, \\ College of Medicine, University of \\ Basra, Basra, Iraq
}

\begin{abstract}
Tinea incognito is a variant of dermatophyte infection of the skin modified by an erroneously applied topical or systemic steroid. Aim of the study: to describe the various clinic-epidemiological aspects of tinea incognito found among our patients. A prospective study was carried out in the Department of Dermatology, Basra Teaching Hospital, Basra, Iraq. The clinical diagnosis was confirmed by mycological tests. Clinical data were described in more detail and the lesions were classified according to the site, shape, and extent. Ninety cases of tinea incognito were seen, median age was 34years. The initial inaccurate diagnoses were eczema in 60 patients $(67 \%)$, intertrigo in $16(18 \%)$ and psoriasis in $14(15 \%)$ patients. The type of topical drugs applied was topical corticosteroid (potent and fluorinated) in most cases (54.4\%), fixed drug combination creams in $36.6 \%$ and a topical calcineurin inhibitor in $5.5 \%$. Commonly presented as acute eczema-like, on hands and trunk, discoid lupus erythematosus-like lesions on the face and psoriasiform lesions on the scalp. Due to the wide range of clinical manifestations, tinea incognito imitates many skin diseases and should, therefore, be considered in any chronic, erythematous, scaly skin lesions not responding to topical treatment.
\end{abstract}

\section{Introduction}

Tinea incognito is a type of ringworm infection modified by corticosteroid (systemic or topical) and other immunosuppressive agents. Corticosteroid is prescribed for pre-existing disease or given mistakenly for the treatment of misdiagnosed tinea. The history is quite characteristic; the patient is often initially satisfied with the treatment, but the eruption relapses, with varying rapidity once the drug is stopped. ${ }^{1}$ The clinical manifestation of tinea incognito varies greatly and can mimic that of various skin diseases such as Systemic Lupus Erythematosus (SLE), eczema, purpura, seborrhoic dermatitis, lichen planus, contact dermatitis, psoriasis and erythema migrans. $^{2-4}$ This characteristic makes its diagnosis difficult and can easily lead to misdiagnosis, preventing prompt an appropriate treatment. ${ }^{5}$ Tinea incognito is a common pitfall in dermatological practice $\&$ in recent years, we noticed a remarkable increase in the prevalence of the disease in both private and hospital settings. The current study aimed to identify various clinicoepidemiological criteria of tinea incognito in our locality.

\section{Materials and Methods}

The present study was a prospective observational study conducted in the Department of Dermatology- Basra Teaching Hospital over a three-year period (2015-2018). A total of 90 patients with tinea incognito were recruited. A detailed history was obtained including, age, sex and duration of the disease, any associated systemic disease, the initial diagnosis of the skin disease, the type of topical medications used previously. A thorough clinical examination was performed to determine the site, extent of distribution of the disease, and describing the type of the lesions. The diagnosis was suspected clinically when the initial lesions were improved partially with topical applications but recurred with even greater virulence once the drugs were stopped. Confirmation of the diagnosis was made by demonstration of fungal elements on direct microscopical examination of $\mathrm{KOH} \mathrm{30 \%} \mathrm{mount} \mathrm{materials.} \mathrm{If} \mathrm{the} \mathrm{hyphae}$ and spores were not detected, the test was repeated after three days. Patients who yielded negative results for a second time were excluded from the study. After the diagnosis was made, treatment with Gresiofulvin in a dose of $20 \mathrm{mg}$ per $\mathrm{kg}$ was administered orally in a single dose at night after eating. The patients were re-examined both clinically and microscopically one month later. The study protocol was approved by the Ethical Committee of Basra College of Medicine/ Basra University and informed consent was obtained from all participants.

\section{Results}

During the three-year period of observation (from 2015 to end the of 2018), a total of 90 cases of tinea incognito were reported. Their ages ranged from 2 to 75 years (median=34years), $58(64 \%)$ of cases were females \& 32 (36\%)were males. The duration of the suffering ranged from 2 weeks to 6 months (median $=4$ weeks). The initial inaccurate diagnoses of skin lesions
Correspondence: Samer A Dhaher, Department of Medicine, College of Medicine, Albradhyia 661031, Basra, Iraq Tel.: +9647801081598

E mail: sameralamir2@yahoo.com

Key words: tinea; incognito; imitator.

Acknowledgements: the author would like to thank all the medical \& paramedical staff at the Department of Dermatology- Basra Teaching Hospital for their help \& support throughout the study.

Conflict of interests: The author declares no potential conflict of interests.

Consent to partecipate: Written informed consent was obtained from the patient.

Ethical approval: the study was approved by the institutional Ethical Committee of College of Medicine/ Basra University (approval number: 004-2015)

Received for publication: 24 September 2019. Accepted for publication: 23 May 2020.

This work is licensed under a Creative Commons Attribution-NonCommercial 4.0 International License (CC BY-NC 4.0).

${ }^{\circ}$ Copyright: the Author(s), 2020

Licensee PAGEPress, Italy

Dermatology Reports 2020; 12:8323

doi:10.4081/dr.2020.8323

were different types of eczema in majority of cases ( 60 patients, $67 \%$ ), intertrigo in 16 cases $(18 \%)$ and psoriasis in 14 cases (15\%). The type of topical drugs applied were: topical corticosteroid (potent and fluorinated) in most of the cases $(49,54.4 \%)$, fixed drug combination creams containing a steroid, an antifungal, and an antibacterial agent in 33(36.6\%), topical calcineurin inhibitor (tacrolimus ointment) in 5 cases (5.5\%) and a topical Calcipotriol/ betamethasone cream or lotion in 3 cases (3.3\%) (Table 1).

The sites of involvement were the trunk and limbs in 29 cases (33\%), the groins in 21 cases $(24 \%)$, the hands in 20 cases (22\%), the face in 11 cases $(13 \%)$, the scalp in 4 cases $(4 \%)$, the foot in 4 cases $(4 \%) \&$ the nail in 2 cases $(2 \%)$.

Clinically, tinea incognito presents in a wide range of manifestations closely mimicking a number of other skin diseases. The most frequent presentations in our study were: i) acute eczema-like lesions with worsening acuteness. These lesions involved more itching and burning, more redness, scales and exudate (Figure 1). this was commonly seen on the trunk and the 
limbs; ii) Tinea imbricata-like lesions, in successive waves of concentric rings of scales and erythema. This was seen mainly on the trunk; iii) Papulo-nodular and bruiselike lesions with dusky red erythema; iv) Atopic eczema-like lesions: this type was observed among infants with scaly erythematous patches on the malar area of the face; v) Discoid lupus erythematosus-like with erythematous polycyclic scaly plaques on the face (Figure 2); vi) Psoriasiform lesions with silvery scale that were thick and piled up on a well-circumscribed red plaque (Figure 3); vii) Chronic hand eczema-like lesions that were erythematous, pruritic and scaly; viii) Napkin dermatitis-like lesions with an erythematous scaly rash in the diaper area (Figure 4).

Considering the response to treatment, most of our patients were successfully treated with oral Gresiofulvin in the recommended daily doses according to body weight. In 4 to 6 weeks, they showed remarkable improvement (Figure 5).

\section{Discussion}

Over the last three years, we were able to see a large number of cases of tinea incognito in both private and hospital settings, with a diversity of clinical presentations. Undisputedly, there has been an increase in the frequency of tinea incognito in our community over the last few years. This may be correlated with malpractice in the field of dermatology, where there was a misdiagnosis of initial fungal infections and, therefore incorrect prescription of topical immunosuppressive agents by unaware physicians. Initially, these medications suppress the normal cutaneous immune responses to dermatophyte, thus enhancing the development of fungal infections. ${ }^{6,7}$ In our study, the majority of patients were females (64\%), while $36 \%$ were males. Despite the previous reporting that the infection rate of dermatophytic fungus was higher in males as compared to females, ${ }^{8}$ Iraqi women were more likely to misuse topical steroids than men. This finding was in contrast to other studies..$^{2,10-14}$ Besides the misapplication of topical fluorinated steroid in most cases, a preparation containing a mixture of potent steroids with antifungal and antibacterial agent (mixed drug combinations) was also used. In the latter case, the potent steroid overcomes the effect of the anti-fungal agent, thus exacerbating superficial dermatophytoses. ${ }^{9}$ Such combinations are irrationally sold as an over the counter medications without any prescription. Similarly, an outbreak of tinea incognito in

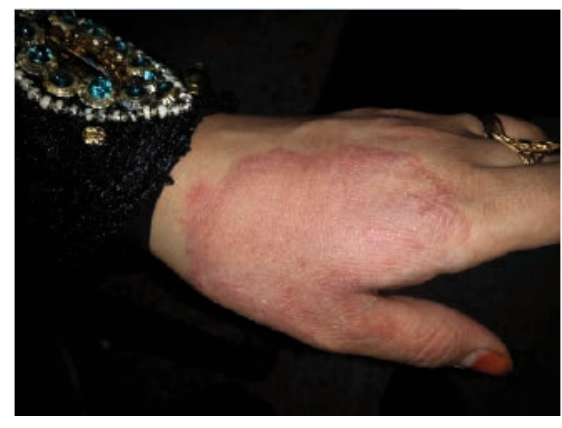

Figure 1. Tinea incognito involving the hands and nails masqueraded as acute or chronic eczema.

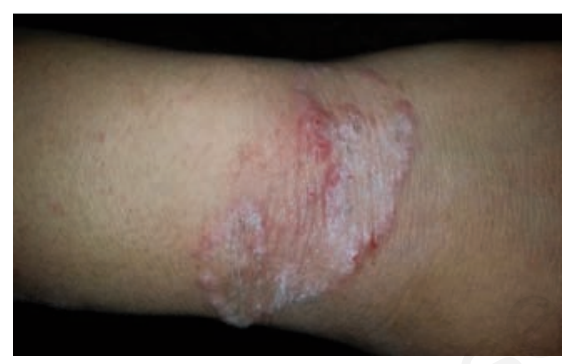

Figure 2. Tinea incognito resembling psoriasis.

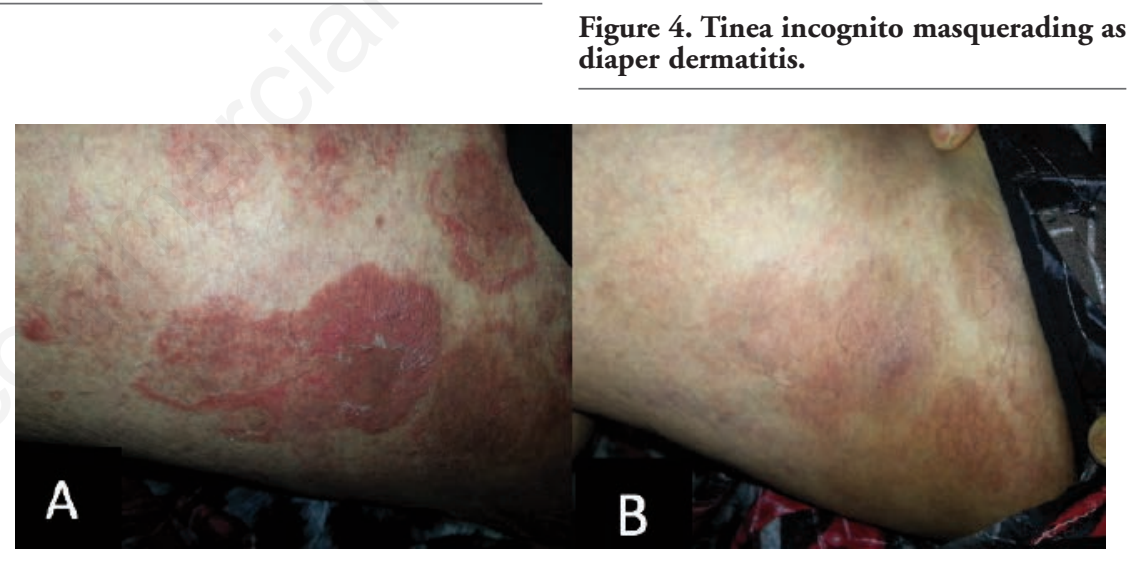

Figure 5. Tinea incognito of groin before (A) and 2weeks after(B) oral Gresiofulvin, showing remarkable improvement.

Table 1. Demographic criteria of patients with tinea incognito

\begin{tabular}{lc} 
Parameters of 90 patients & No (\%) \\
Age (years) & $2-75$ (median 34) \\
Gender & Females:58(64), males:32(36) \\
\hline Duration & 2 wks-6 months(median:4wks) \\
Initial inaccurate diagnosis & \\
- Various types of eczema & $60(67)$ \\
- Intertrigo & $16(18)$ \\
- Psoriasis & $14(15)$ \\
\hline Type of topical drugs & \\
- Corticosteroids & $49(54.4)$ \\
- Fixed drug combinations & $33(36.6)$ \\
- Calcineurin inhibitors & $5(5.5)$ \\
- Calcipotriol/ betamethasone & $3(3.3)$ \\
\hline
\end{tabular}

Figure 3. Tinea incognito on the face resembling lupus erythematosus.

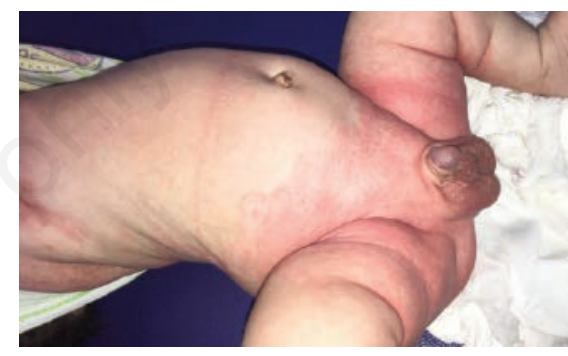

Figure 4. Tinea incognito masquerading as diaper dermatitis.$$
\text { . }
$$

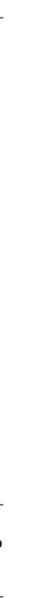


India was reported by Verma et al. following improper application of these topical combinations. ${ }^{11}$ Recently, misuse of topical calcineurin inhibitors has increased and in $5.5 \%$ of our cases. This was followed the application of these drugs. This malpractice not only resulted in an increase in the prevalence of tinea incognito, but it also warrants a redefinition of the disease itself as a subtype of dermatophyte infection caused by erroneous use of systemic/topical corticosteroids or topical calcineurin inhibitors. ${ }^{10}$ It worth noting that we did not encounter cases of tinea incognito that were caused by systemic steroids or immunosuppressive agents. Other than nail infection, tinea corporis was the most frequent dermatophyte infection in Iraq. ${ }^{8}$ We found that tinea corporis incognito was also the most prevalent subtype and in many patients with a presentation that was widespread and extensive. Based on both our own observations and on reports of other retrospective studies, , $^{2,10,14}$ tinea incognito has a diverse morphological appearance and clinically, it may imitate a long list of skin diseases such as psoriasis, acute or chronic eczema, or SLE. Therefore, we can propose the term "the new imitator" for tinea incognito, making it the third of such well-known imitators (with the other two being, drug eruptions and syphilis). However, in many instances, the lesions of tinea incognito were distinguished by their retained tendency for centrifugal extension with hyperactivity at the border. This can call the attention to the correct diagnosis. Even more, lesions on the hands, in particular, may show marches toward the nails, causing nail destruction. Therefore, eczema- like lesions on hands with nail involvement should be carefully evaluated to rule out tinea incognito. Involvement of the scalp was seen in four cases; all of these were children erroneously diagnosed with psoriasis. These children were on long-term use of topical steroids with or without cal- cipotriene which caused not only local spread but also hair shedding and non- scarring alopecia. It is known that the application of topical steroids to a dermatophyte scalp infection may decrease the local immunological host reaction, allowing the underlying dermatophyte infection to persist or, even acquire the ability to invade deeper tissues and hair follicles. ${ }^{13,15}$

\section{Conclusion}

Tinea incognito is a common problem in the Iraqi population. In a clinical perspective, the disease can imitate a long list of other known skin diseases. Due to a wide variety of presentations, tinea incognito should be taken into account in any chronic, erythematous, scaly lesions that are unresponsive to topical treatments. Misprescribed drugs other than corticosteroids, like calcineurin inhibitors should also be considered as a possible cause.

\section{References}

1. Hay RJ, Ashbee HR. Mycology. In: Burns T, Breathnach S, Cox N, Griffiths Ch (editors). Rook's Textbook of Dermatology. $8^{\text {th }}$ ed. Oxford, UK: Blackwell, 2010.

2. Romano C, Maritati E, Gianni C. Tinea incognito in Italy: a 15-year survey. Mycoses 2006;49:383-7.

3. Agostini G, Knopfel B, Difonzo EM. Universal dermatophytosis (tinea incognito) caused by Trichophyton rubrum. Hautarzt 1995;46:190-3.

4. Guenova E, Hoetzenecker W, Schaller M, Rocken M, Fierlbeck G. Tinea incognito hidden under apparently treatment-resistant pemphigus foliaceus.
Acta Derm Venereol 2008;88:276-7.

5. Feder HM. Tinea incognito misdiagnosed as erythema migrans. New Engl J Med 2000;343:69.

6. Solomon BA, Glass AT, Rabbin PE. Tinea incognito and "over-the-counter" potent topical steroids. Cutis 1996;58:295-6.

7. Siddalah N, Erickson O, Miller G, Elston DM. Tacrolimus induced Tinea incognito. Cutis 2004;73:237-8.

8. Abed Ali F, Jawad K. Al-Janabi A, Alhattab M. Prevalence of dermatophyte fungal infection in Hillah, Iraq. Int J Chem Tech Res 2017;10:827-37.

9. Jowkar F, Saki N, Derayesh M, Aslani FS. Tinea incognito simulating dermatitis herpetiformis: An unusual case report. Iran J Dermatol 2016;19:54-6.

10. Kim W, Tae-Wook Kim T, Je-Ho Mun J, Song M, Kim HS. Tinea Incognito in Korea and Its Risk Factors: Nine-Year Multicenter Survey. J Korean Med Sci 2013;28:145-51.

11. Verma S, Madhu R. The great Indian epidemic of superficial dermatophytoses: An appraisal. Indian J Dermatol 2017;62:227-36

12. Al-Dhalimi M, Aljawahiry N. Misuse of topical corticosteroids: a clinical study in an Iraqi hospital. E Mediterr Health J 2006;12:847-52.

13. Degreef H, Doncker P(editors). Fighting Fungal Infection Around the Globe: Itraconazole in Perspective. Kent, UK: Wells Medical Holdings, 2000.

14. Ansar A, Fershchian M, Naseri H, Ghiasian SA. Clinico-epidemiological $\&$ mycological aspects of tinea incognito in Iran: a 16-years study. Med Mycol J 2011;52:25-32.

15. Erbagci Z. Topical therapy for dermatophytoses: should corticosteroids be included? Am J Clin Dermatol 2004; $5: 375-84$ 